\title{
Low fifth minute Apgar scores and its associated factors among newborns in public health facilities of Arba Minch town, southern Ethiopia: a cross- sectional study
}

Abera Mersha ( $\sim$ mershaabera@gmail.com )

Arba Minch University https://orcid.org/0000-0002-4366-4128

Shitaye Shibiru

Arba Minch University

Agegnehu Bante

Arba Minch University

\section{Research}

Keywords: Apgar scores, Newborns, Arba Minch

Posted Date: May 7th, 2020

DOI: https://doi.org/10.21203/rs.3.rs-26429/v1

License: (c) (i) This work is licensed under a Creative Commons Attribution 4.0 International License.

Read Full License 


\section{Abstract}

Background: Low Apgar scores in the childbirth period increased risk globally and significantly contributes to both newborn morbidity and mortality. Hence, it is very essential to update information on the status of low fifth-minute Apgar scores and factors affecting. The majority studies conducted were retrospective and prone to bias. In addition, there is limited study in Ethiopia that shows the recent status of low fifth-minute Apgar scores and factors affecting. Therefore, this study intends to fill those gaps.

Methods: A facility-based cross-sectional study conducted among 286 newborns in public health facilities of Arba Minch town, southern Ethiopia from February 6 to March 9, 2019. A study participants selected by using systematic random sampling method. Pre-tested interviewer-administered questionnaires and checklist used to collect the data. Data entered into Epi data version 3.1 and exported to Stata version 15 for analysis. A crude and adjusted odds ration computed in the binary logistic regression model. In this study, P-value, $<0.05$ considered to declare factors as statistically significant.

Results: In this study, $17.8 \%$ (95\% Cl: $13.8 \%, 22.7 \%)$ of neonates had low fifth-minute Apgar scores. Condition of labor (induced/augmented) ( $\mathrm{AOR}=3.33,95 \% \mathrm{Cl}$ : 1.24, 8.90), meconium-stained liquor (AOR=3.37, 95\% Cl: 1.17, 9.74), and birth weight (AOR=3.48, 95\%Cl: $1.23,9.86)$ were significantly associated with neonate's low fifth-minute Apgar scores.

Conclusions: This study indicated that a significant number of newborns resulted in low fifth-minute Apgar scores. Strengthen the provision of health information during antenatal care; avoid delay in screening high-risk mothers during pregnancy and delivery, and give immediate interventions should recommended. Keywords: Apgar scores, Newborns, Arba Minch

\section{Background}

The Apgar scores is a simple statistical model of five physiological signs, devised by Dr. Virginia. Apgar in 1952 to decipher immediate neonatal wellbeing as recently reviewed by using clinical assessment tool newborn infants evaluated based on five variables: Heart rate, Respiratory effort, Muscle tone, Reflex


have used as an indicator of "baby with a problem". Ever since Virginia Apgar devised tool in 1952 as "a means of evaluating the physical condition of the newborn immediately after birth"[3]. Besides, neonates whose fifth-minutes Apgar score lessen seven considered as having parental asphyxia[4].

Low Apgar scores in the childbirth period increased risk globally and significantly contributes to both newborn morbidity and mortality $[1,5,6]$. Evidence showed a consistent association of low Apgar scores with increased risks of neonatal and infant death and with a neurologic disability, including cerebral palsy, epilepsy, and cognitive impairment [7-11].

A pocket of studies showed low Apgar scores contributed $13.6 \%$ of causes of neonatal morbidity and mortality $[4,12]$. The rate of deaths due to perinatal asphyxia is approximately 10 times that of developed 
countries though accurate estimates of neurodevelopment sequels from asphyxia in sub-Saharan Africa are not available likely that these sequel are many times more prevalent in developing countries in developed countries[13].

Studies conducted in West and sub-Saharan, and North African showed the percentage of receiving a low Apgar score was $3.9 \%$, and $1.8 \%$ respectively [14]. Besides, a study conducted in North-west, Ethiopia reported $13.8 \%[15]$. Studies stated that the most determinate factors associated with low fifth-minute Apgar scores were non-vertex fetal presentation, prolonged labor, presence of meconium-stained liquor, induced/augmented labor, low birth weight, gestational age, multiple pregnancies, previous cesarean section, and body mass index [15-18].

As stated above a few studies conducted in different parts of the world that showed the status of low fifth-minute Apgar scores [14, 15]. However, the majority of them were retrospective and prone to bias. In addition, there is limited study in Ethiopia that shows the recent status of low fifth-minute Apgar scores and factors affecting. Therefore, the main of this study was to fill those gaps and show the recent status of low fifth-minute Apgar scores and its associated factors in public health facilities of Arba Minch town, southern Ethiopia.

\section{Methods}

\section{Study setting, design, and period}

This institution-based cross-sectional study conducted in public health facilities of Arba Minch town, southern Ethiopia from February 6 to March 9, 2019. Arba Minch town is the capital of Gamo Zone, which is $505 \mathrm{~km}$ away from Addis Ababa and $275 \mathrm{~km}$ from Hawassa. There are there three public health facilities in Arba Minch town (Arba Minch general hospital, Secha health center, and Sikela health center). Arba Minch general hospital services inpatient and outpatient service. It has total of 470 beds and more than 400 working staffs. Secha health center opened in 2012; situated plain surface found in Arba Minch town. There are more than 51 working staffs and the health center provides delivery services for 24 hour a day for 7 day a week. Sikela health center started delivering health care services to the public since 1966. There are total of 78 staff working in the health center with different shift. The health center provides delivery services for 24 hour a day for 7 days a week.

\section{Population and eligibility criteria}

All live births at public health facilities of Arba Minch town were source population, and those during the study period were study population for this study. All mothers whose age $\geq 18$ years old with singleton live births were involved, and those newborns with gross congenital malformation excluded from this study.

\section{Sample size determination and sampling procedure}


A separate sample size calculated for each objective in Epi info7 Stat Calc. The assumptions were a proportion of low fifth-minute Apgar scores (13.8\%) from the study conducted in North-west, Ethiopia[15], $95 \% \mathrm{Cl}, 4 \%$ margin of error, and a $5 \%$ non-response rate. Based on the stated assumptions, the calculated sample size for this study was 301 . A systematic random sampling method used to reach each study participant. The determined sample size achieved by taking monthly average deliveries from preceding year-registered data.

\section{Data collection methods}

A structured interviewer-administered pre-tested questionnaire and checklist to review the medical records used to collect the data. The tools were adapted from different works of literature (Additional file 1). Welltrained data collectors assigned to collect the data from the delivery ward. The data collectors gave information about the study aim and the possible procedures for study participants before interviewing and abstracting the medical records.

\section{Variables}

Low fifth-minute Apgar scores was dependent variable and socio-demographic characteristics, obstetric, and neonatal factors were independent variables for this study.

\section{Measurements}

Apgar scores computed by using five components. Appearance: pink (2 points), bluish extremities (1 point), and blue ( 0 points). Pulse rate: $\geq 100$ beats/minute ( 2 points), $<100$ ( 1 point), and none ( 0 points). Grimace: crying ( 2 points), whimpering (1 point), and silence ( 0 points), Activity: active ( 2 points), moderate ( 1 point), and limp ( 0 points). Respiration: regular breathing ( 2 points), irregular and $<30$ breath/minute (1 point), and none ( 0 points). Then the score of each finding summed and a score below value seven at fifth-minute assessment considered as low fifth-minute Apgar scores $[3,15,19]$.

\section{Data quality control}

To ensure quality, the questionnaire translated into the local language by verified translators. The questionnaires pre-tested in the hospital with a similar status. Data checked for completeness, accuracy, clarity, and consistency before entered into the software. Proper coding and categorization of data maintained for the quality of the data.

\section{Data analysis and processing}

The data coded, cleaned, edited and entered into Epi data version 3.1 and then exported to Stata version 15 for analysis. Descriptive analysis done by computing proportions, and summary statistics. Binary logistic regression used to see the association between each independent variable and the outcome variable. The log-likelihood ratio (LR) used to test the goodness of fit. All variables with $P<0.2$ in the bivariate analysis were included in the final model of multivariable analysis. The standard error of $\geq 2$ 
considered for the existence of multi co-linearity. The crude odds ratio (COR) and adjusted odds ratio (AOR) along with 95\% confidence interval $(\mathrm{Cl})$ estimated. In this study, P-value, $<0.05$ considered to declare a result as a statistically significant association. Then the information presented by using simple frequencies, summary measures, tables, and figures.

\section{Results}

\section{Maternal socio-demographic and obstetric characteristics}

In this study, 286 study participates involved, which gave a response rate of $95 \%$. The mean and standard deviation of the age of mothers was (25.79 \pm 4.14$)$. Married respondents constituted $268(93.7 \%)$, and Orthodox Christian religion followers had 134 (46.9\%). Of the participants, 230 (80.40) had a family size of three to five, and 193 (67.5\%) were urban dwellers. One hundred seventy-one (59.8) of the mothers were multigravida. Two hundred eighty $(97.9 \%)$ of the mothers had antenatal care during the index pregnancy, and from them, 204 (72.9\%) were four or more visits. Only one $(0.3 \%)$ of the mother faced amniotic fluid disorder pregnancy, three (1.0\%) had a fever during labor time, and two $(0.7 \%)$ had a history of neonatal deaths. Out of the study participants, four (1.4\%) were chronic diseases, and three (1.0\%) had a history of bleeding during pregnancy (antepartum hemorrhage) (Table 1).

\section{Factors associated with the neonate's low fifth-minute Apgar scores}

This study founded that, $17.8 \%$ (95\% Cl: $13.8 \%, 22.7 \%)$ of neonates had low fifth-minute Apgar scores. In the multivariable model, condition of labor (induced/augmented), meconium-stained liquor, and birth weight were significantly associated with neonate's fifth-minute Apgar scores. Mothers who induced/augmented during labor were 3.33 times, and who had meconium-stained liquor were 3.37 times more likely gave neonates with low fifth-minute Apgar scores (AOR=3.33, 95\% Cl: 1.24, 8.90) and $(A O R=3.37,95 \% \mathrm{Cl}: 1.17,9.74)$ respectively. The odds of low fifth-minute Apgar scores were 3.48 among neonates whose birth weight had less than $2500 \mathrm{~g}(\mathrm{AOR}=3.48,95 \% \mathrm{Cl}: 1.23,9.86)$ (Table 2).

Table 1: Maternal socio-demographic, obstetric, and neonatal characteristics in public health facilities of Arba Minch town, southern Ethiopia, 2019( $n=286)$ 


\begin{tabular}{|c|c|c|}
\hline Variables & Frequency & Percentage \\
\hline \multicolumn{3}{|l|}{ Age } \\
\hline $18-34$ & 270 & 94.4 \\
\hline$\geq 35$ & 16 & 5.6 \\
\hline \multicolumn{3}{|l|}{ Educational status } \\
\hline No formal education & 16 & 5.6 \\
\hline Grade 1-8 & 114 & 39.9 \\
\hline Grade $9-12$ & 85 & 29.7 \\
\hline College and above & 71 & 24.8 \\
\hline \multicolumn{3}{|l|}{ Occupation of mother } \\
\hline Government employer & 28 & 9.8 \\
\hline Merchant & 97 & 33.9 \\
\hline Private worker & 45 & 15.7 \\
\hline Housewife & 111 & 38.8 \\
\hline Other* & 5 & 1.7 \\
\hline \multicolumn{3}{|l|}{ Condition of labor } \\
\hline Spontaneous & 251 & 87.8 \\
\hline Induced/Augmented & 35 & 12.2 \\
\hline \multicolumn{3}{|l|}{ Duration of labor } \\
\hline$<12$ hour & 161 & 56.3 \\
\hline$\geq 12$ hour & 125 & 43.7 \\
\hline \multicolumn{3}{|c|}{ Duration of rupture of membrane before the onset of labol } \\
\hline$<18$ hour & 243 & 85.0 \\
\hline$\geq 18$ hour & 43 & 15.0 \\
\hline \multicolumn{3}{|l|}{ Mode of delivery } \\
\hline SVD & 240 & 83.9 \\
\hline CS & 46 & 16.1 \\
\hline \multicolumn{3}{|l|}{ Fetal presentation } \\
\hline Vertex & 240 & 83.9 \\
\hline
\end{tabular}




\section{Meconium-stained liquor}

\begin{tabular}{lll} 
Yes & 25 & 8.7 \\
\hline No & 261 & 91.3 \\
\hline
\end{tabular}

\section{Hypertensive disorder during pregnancy}

\begin{tabular}{lll} 
Yes & 30 & 10.5 \\
\hline No & 256 & 89.5
\end{tabular}

\section{Gestational age}

$\begin{array}{lll}<37 \text { week } & 52 & 18.2 \\ \geq 37 \text { week } & 234 & 81.8\end{array}$

\section{Birth weight}

$\begin{array}{lll}<2500 \mathrm{~g} & 60 & 21.0 \\ \geq 2500 \mathrm{~g} & 226 & 79.0\end{array}$

*Daily laborer and student, SVD: spontaneous vaginal delivery, and CS: cesarean section

Table 2: Factors associated with the neonates low fifth-minute Apgar scores in public health facilities of Arba Minch town, southern Ethiopia, 2019(n=286) 
Variables

Low fifth-minute Apgar scores $\quad(95 \% \mathrm{Cl})$

$\begin{array}{llll}\text { Yes } & \text { No } & \text { Crude OR } & \text { Adjusted OR }\end{array}$

\section{Gravidity}

Primigravida

$21(18.3 \%) \quad 94(81.7 \%)$

$1.05(0.57,1.94)$

$1.18(0.53,2.65)$

Multigravida

$30(17.5 \%)$

$141(82.5 \%)$

1

1

Fetal presentation

\begin{tabular}{lllll} 
Vertex & $31(12.9 \%)$ & $209(87.1 \%)$ & 1 & 1 \\
\hline Non-vertex & $20(43.5 \%)$ & $26(56.5 \%)$ & $5.19(2.59,10.38)$ & $2.41(0.80,7.24)$
\end{tabular}

Meconium-stained liquor

$\begin{array}{lllll}\text { Yes } & 12(48.0 \%) & 13(52.0 \%) & 5.25(2.23,12.36) & 3.37(1.17,9.74)^{*} \\ \text { No } & 39(14.9 \%) & 222(85.1 \%) & 1 & 1\end{array}$

Condition of labor

\begin{tabular}{lllll} 
Spontaneous & $34(13.5 \%)$ & $217(86.5 \%)$ & 1 & 1 \\
\hline Induced/Augmented & $17(48.6 \%)$ & $18(51.4 \%)$ & $6.03(2.85,12.83)$ & $3.33(1.24,8.90)$ * \\
Mode of delivery & & & & 1 \\
\hline SVD & $32(13.3 \%)$ & $208(86.7 \%)$ & 1 & $1.77(0.59,5.32)$ \\
CS & $19(41.3 \%)$ & $27(58.7 \%)$ & $4.57(2.28,9.16)$ &
\end{tabular}

Gestational age

$\begin{array}{lllll}<37 \text { week } & 23(44.2 \%) & 29(55.8 \%) & 5.83(2.97,11.46) & 2.39(0.79,7.32) \\ \geq 37 \text { week } & 28(12.0 \%) & 206(88.0 \%) & 1 & 1\end{array}$

\section{Birth weight}

$\begin{array}{lllll}<2500 \mathrm{~g} & 29(48.3 \%) & 31(51.7 \%) & 8.67(4.44,16.96) & 3.48(1.23,9.86)^{\star} \\ \geq 2500 \mathrm{~g} & 22(9.7 \%) & 204(90.3 \%) & 1 & 1\end{array}$

Hypertensive disorder during pregnancy

$\begin{array}{lllll}\text { Yes } & 12(40.0 \%) & 18(60.0 \%) & 3.71(1.66,8.31) & 1.88(0.60,5.85) \\ \text { No } & 39(15.2 \%) & 217(84.8 \%) & 1 & 1\end{array}$

\section{Duration of labor}

$\begin{array}{lllll}<12 \text { hour } & 26(16.1 \%) & 135(83.9 \%) & 1 & 1 \\ \geq 12 \text { hour } & 25(20.0 \%) & 100(80.0 \%) & 1.29(0.71,2.38) & 0.91(0.39,2.11)\end{array}$


Duration of rupture of membrane before the onset of labor

$\begin{array}{lllll}<18 \text { hour } & 37(15.2 \%) & 206(84.8 \%) & 1 & 1 \\ \geq 18 \text { hour } & 14(32.6 \%) & 29(67.4 \%) & 2.69(1.29,5.56) & 1.51(0.55,4.11)\end{array}$

* Significant at $P$-value<0.05

\section{Discussion}

The overall status of low fifth-minute Apgar scores among singleton newborns was $17.8 \%(95 \% \mathrm{Cl}: 13.8 \%$, $22.7 \%$ ). This study identified that condition of labor (induced/augmented), meconium-stained liquor, and birth weight were significant factors associated with neonate's low fifth-minute Apgar scores.

The magnitude of low fifth-minute Apgar scores in this finding was higher than studies conducted in West and sub-Saharan African (3.9\%), North African (1.8\%)[14], resource-constrained settings (2.3\%)[13], and a study conducted in North-west Ethiopia (13.8\%)[15]. The reason for this difference related to socioeconomic status, health-seeking behavior of pregnant mothers, socio-cultural factors, and methodological aspects (study setting, period, design, and measurements).

In this study, condition of labor (induced/augmented) and meconium-stained liquor had a significant association with low fifth-minute Apgar scores. This is congruent with a study conducted in North-west, Ethiopia[15]. This is in fact that induction/augmentation indicated to shorten labor for the mother with prolonged labor. Consequently, the progress of labor, as well as the drug administered for that, hurts the fetus that results for low fifth-minute Apgar scores. In addition, meconium-stained liquor is one of the indications of fetal distress and other intrapartum complications, and result for aspiration. Therefore, those newborns are most likely has low fifth-minute Apgar scores. As showed by this survey, low birth weight $(<2500 \mathrm{~g}$ ) were significantly associated with low fifth-minute Apgar scores. This is in line with studies conducted in Brazil, and North-west, Ethiopia[15, 20]. The reason for this is that, lowest birth weight associated with prenatal complications. These complications result in adverse fetal and neonatal outcomes, such as birth asphyxia, low Apgar scores, stillbirth, early and late neonatal mortality, and other morbidities.

Fetal presentation and duration of labor were not significantly associated with low fifth-minute Apgar scores. This is inconsistent with a study done in North-west, Ethiopia[15]. The inconsistency may be due to differences in methodological aspects, and the health care delivery system (accessibility, and availability of nearby health facilities).

The main limitation of the study was that the study might subjected to recall bias. The causal association was under caution as the study design was cross-sectional. 


\section{Conclusions}

This study indicated that a significant number of newborns resulted in low fifth-minute Apgar scores. There are different factors during the continuum of care for pregnant mothers that result in low fifthminute Apgar scores. Therefore, early identification and intervention for those conditions are very vital to improve neonatal health. In general, this study identified that condition of labor (induced/augmented), meconium-stained liquor, and low birth weight $(<2500 \mathrm{~g})$ were independent predictors for low fifth-minute Apgar scores. Based on the above finding the scholars like to provide the following recommendations: Strengthen the provision of health information during antenatal care, avoid delay in screening high-risk mothers, and give immediate interventions for those conditions. The other scholars do further investigation to identify other factors by using other study designs in a large group of the population, typically experimental or follow-up studies.

\section{Abbreviations}

AOR: Adjusted Odds Ratio, CS: Cesarean Section, Cl: Confidence Interval, COR: Crude Odds Ratio, and SVD: Spontaneous Vaginal Delivery

\section{Declarations}

\section{Ethics approval and consent to participate}

Ethical clearance obtained from Arba Minch University, College of Medicine and Health Sciences, Institutional Research Ethics Review Board (IRB). All the study participants informed about the purpose of the study, their right to refuse and written and signed voluntary consent obtained from all study participants before the interview. The respondents were also been told that the information obtained from them was treated with complete confidentiality and do not cause any harm to them.

\section{Consent for publication}

Not applicable

\section{Availability of data and materials}

The data will not share to preserve participant anonymity.

\section{Competing interests}

All authors assert that they have no competing interests

\section{Funding}

No funding provided for this research project from any organization 


\section{Authors' contributions}

AM designed the study, involved in data collection, done analysis and interpretation of the result and drafted the paper and participated in preparing all versions of the manuscript. SS and AB assisted in the design and the proposal development, monitored data collection, assisted during analysis and revised subsequent drafts of the paper. All authors read and approved the final manuscript.

\section{Acknowledgments}

My unreserved thank go to the Chief Executive Officer of public health facilities of Arba Minch town for allowed to do this study in that institution, data collectors, and study participants. Finally, yet importantly, I would like to say thank you for all peoples who support directly or indirectly

\section{Authors' information}

Abera Mersha (BScN, MSc in Maternity and Neonatal Nursing), School of Nursing, College of Medicine and Health Sciences, Arba Minch University, Arba Minch, Ethiopia.

Shitaye Shibiru (BScN, MSc in Maternity and Reproductive Health Nursing), School of Nursing, College of Medicine and Health Sciences, Arba Minch University, Arba Minch, Ethiopia.

Agegnehu Bante (BScN, MSc in Maternity and Neonatal Nursing), School of Nursing, College of Medicine and Health Sciences, Arba Minch University, Arba Minch, Ethiopia.

\section{References}

1. Apgar VA. Proposal for a new method of evaluation of the newborn infant. Current Research Anesth Analg, 1953. 32: 260-267.

2. Ondoa-Onama C, Tumwine J. Immediate outcome of babies with a low Apgar score in Mulago Hospital, Uganda. East African Medical Journal, 2003. 80:1.

3. Papile L. Apgar Score in the 21st Century, Ed. New England Medical Journal, 2001. 344: 519-520.

4. Lagatta J, Yan K, and Hoffmann R. The association between 5-min Apgar score and mortality disappears after 24 hour at the borderline of viability. Acta paediatrica (Oslo, Norway : 1992, 2012. 101(6): e243-e247.

5. Lawn JE, Lee Ac, Kinney $M$ et al.Two million Intrapartum stillbirth and neonatal deaths: where, why and what can we do? Int J Gyn Eco obstetrics, 2009:107:55-519.

6. Lawn J, Shibuya K, steinc. No cry at birth: global estimates of Intrapartum stillbirths and Intrapartumrelated neonatal deaths. Bull WHO, 2005. 83(6):409-17.

7. Ehrenstein V, Pedersen L, Grijota M et al. Association of Apgar score at five minutes with a long-term neurologic disability and cognitive function in a prevalence study of Danish conscripts. BMC Pregnancy and Childbirth, 2009. 9:14. 
8. Stuart A, Otterblad Olausson P, and Källen K. Apgar Scores at 5 Minutes After Birth in Relation to School Performance at 16 Years of Age. Obstetrics \& Gynecology, 2011. 118:2.

9. Andrea S, Petra OO, Kallenk K. Apgar Scores at 5 Minutes After Birth in Relation to School Performance at 16 Years of Age. The American College of Obstetricians and Gynecologists. Published by Lippincott Williams \& Wilkins, 2011. 118(2):201-204.

10. Harrington DJ, Redman CW, Mowden $M$ et al. The Long term outcome in surviving Infant, with Apgar zero at 10 minutes: a systematic review of the literature and hospital-based cohort. American Journal of Obstetrics \& Gynecology, 2007. 196:463el-5.

11. Herrnstein V, Pedersenal Grijota $\mathrm{M}$ et al. Association of Apgar score at five minutes with long term neurologic disability and cognitive function in a prevalence study of Danish conscripts. BMC Pregnancy Childbirth, 2009. 9:14.

12. Paul VK, Singh M, Sundaram KR. et al. Correlates of mortality among hospital - born neonates with birth asphyxia. Natl. Med. J. India, 1997. 10:54-57.

13. Olusanya BO, Solanke OA. Correlates of birth asphyxia using two Apgar score classification methods. Nig Q J Hosp Med, 2010. 20(4):153-61.

14. Cacciani L, Asole S, Polo A, Franco F, Lucchini R, De Curtis M, Di Lallo D, Guasticchi G. Perinatal outcomes among immigrant mothers over two periods in a region of central Italy. BMC Public Health, 2011 May 10;11:294. DOI: 10.1186/1471-2458-11-294. PMID: 21569229; PMCID: PMC3119162.

15. Gudayu TW. Proportion and factors associated with low fifth minute Apgar score among singleton newborn babies in Gondar University referral hospital; North West Ethiopia. African health sciences, 2017. 17(1): 1-6.

16. Jeganathan R, Karalasingam SD, Hussein Julia et al. Factors associated with recovery from 1 minute Apgar score $<4$ in live, singleton, term births: an analysis of Malaysian National Obstetrics Registry data 2010-2012. BMC Pregnancy and Childbirth, 2017. 17(1): 110-110.

17. Svenvik M, Brudin L, and Blomberg M. Preterm Birth: A Prominent Risk Factor for Low Apgar Scores, 2015:8.

18. Assunção S, Eugenia M, Duarte BC, Juliana A et al. Low Apgar scores at 5 minutes in a low-risk population: Maternal and obstetrical factors and postnatal outcome. Revista da Associação Médica Brasileira (English Edition), 2012. 58(5): 587-593.

19. Casey B. The continuing value of the Apgar scores for assessment of Newborn. New England Medical Journal, 2001. 344(7):467-471.

20. Kilsztajn S, de Souza Lopes E, Nunes do Carmo MS, de Andrade AM. Apgar scores associated with mode of delivery in São Paulo State, Brazil. Cad Saude Publica, 2007. 23(8):188692.

\section{Supplementary Files}

This is a list of supplementary files associated with this preprint. Click to download. 
- Additionalfile1.pdf

- Additionalfile2.pdf

Page 13/13 